\title{
Current Situation and Countermeasures of Sports Participation of Heilongjiang Citizens
}

\author{
Xin Li ${ }^{1, ~ a}$, * Jingjing Guan ${ }^{1, b}$ \\ ${ }^{1}$ Qiqihar Medical University, Qiqihar, Heilongjiang, China, 161006 \\ *Corresponding Author: Xin Li \\ aemail:360121833@qq.com, bemail:33094995@qq.com
}

Keywords: Heilongjiang Province, Citizens, Sports Participation

\begin{abstract}
Due to the great pressure of modern society, people in cities are often in the state of sub-health. Sports participation is an effective way to change the health status of residents. In this paper, the current situation of sports participation of citizens in Heilongjiang province was investigated, including the number of people involved, the choice of sports items, activity venues and influencing factors. Based on the investigation, this paper gives the corresponding countermeasures to provide some references for the related researchers.
\end{abstract}

\section{Research Subject and Method}

In this study, we randomly selected 700 city residents of Heilongjiang Province as the research subjects. They come from Harbin, Qiqihar, Mudanjiang, Heilongjiang, Daqing and Heihe, including 329 males and 371 females.

According to the research content and the research purpose,the author searched the Wanfang Data and CNKI network resources as well as the Heilongjiang University and Northeastern University Library. The reading data of a large number of network resources, journals and books and other literature, with the literature data for the study provide valuable theoretical support, in the method as a reference. According to research content and purpose, the author visited the Heilongjiang Province Sports Bureau, Heilongjiang Sports Federation to communicate with them. Based on seeking the social sports science, sports management and school physical education experts, a questionnaire is for residents. According to the purpose and content of this study, the questionnaires adapts multiple-choice questions and answer a single, closed and open the way to answer the questionnaire. They are the basic situation of city residents, city residents to participate in sports activities of the situation and effect of city residents to participate in sports activities. Reliability is a necessary but not sufficient condition for validity. Reliability is the reliability of the test results. It shows the consistency, consistency, reproducibility and stability of the test results. In order to test the validity of the questionnaire, we carried out the test reliability and the reliability met the requirements of this study.

\section{Survey Results and Analysis}

Number of Participants. Sports population is one of the important indicators of the development of sports in a country. As shown in Table 1, Heilongjiang city residents accounted for $53.3 \%$ of the total number of survey participants. The proportion of male participation was $63.8 \%$, the level of participation was significantly higher than the level of female participation. From an objective analysis, the city residents to participate in sports activities is need to have some time as basic conditions, through the understanding of professional condition, now the pressure of work, high work efficiency, makes a lot of employees with the rest of the time relatively few. Most women are in the age of the family, not only to work, but also to bear the work of the home and to take care of children or the elderly. Women's time and energy to participate in sports is far less than 
that of men.

Table 1 Number of participants in the survey

\begin{tabular}{|c|c|c|c|}
\hline Gender & Total & Participants & Percent \\
\hline Male & 329 & 210 & $63.8 \%$ \\
\hline Female & 371 & 163 & $43.9 \%$ \\
\hline Total & 700 & 373 & $53.3 \%$ \\
\hline
\end{tabular}

Choice of Sports Items. Heilongjiang province city residents to participate in sports fitness and the choice of content, the main choice of male residents is walking, jogging, table tennis, badminton, female residents is walking, running, dancing, aerobics bowling etc.. Most older people like fitness project is walking, martial arts and Croquet; middle-aged people like most fitness project is running, walking and Wushu, qigong; young people's favorite projects is the ball; the children's favorite sports is swimming, skating and playing ball. Sports project is an important part of everyone's sports activities. In the urban residents to participate in sports survey found in row in the top five were walking, jogging, badminton, football, square dance, yoga; in different gender selection ranked in the first item of the same, are to walking, running as their sports activities. In different gender groups to participate in sports activities, on the one hand, is bound to be affected by their own psychological and physiological characteristics, but also reflects the "gender culture". In the survey results can be seen, the urban residents of the sports project is still more obvious gender preference.

Table 2Choice of sports items in the survey

\begin{tabular}{|c|c|c|c|}
\hline Gender & First & Second & Third \\
\hline Male & Walk or Run(151) & Badminton(101) & Football(81) \\
\hline Female & Walk or Run(168) & Dance(129) & Yoga(99) \\
\hline
\end{tabular}

Activity Venues. At present, our country has formed a tide of the development of mass sports, the city residents to participate in sports activities place concern. People participate in sports activities in the process of selection of places in the top three is a small square, park, near the school. People will choose to the choice of venue for some of the nearest public locations; row in fourth place is unit of stadium and gymnasium for physical exercise; ranked fifth in the stadium facilities fees, to participate in physical exercise as part of residents income is quite considerable. From this result, it can be seen that the development of public sports facilities is very important to strengthen the awareness of urban residents to enjoy sports facilities for physical exercise, but also an effective way to increase the sports population.

Table 3Activity Venues in the survey

\begin{tabular}{|c|c|c|}
\hline & Frequency & Sequence \\
\hline Residential Square & 321 & 1 \\
\hline Park & 271 & 2 \\
\hline School or Universities & 159 & 3 \\
\hline Sports Facilities of Units & 156 & 4 \\
\hline Sports Stadiums & 87 & 5 \\
\hline Public Spaces & 51 & 6 \\
\hline Others & 38 & 7 \\
\hline
\end{tabular}

Influencing Factors. Influence of Heilongjiang city residents to participate in leisure sports activities of the main factors: facilities, leisure time, habit, physical and mental condition, economic conditions and guide the staff. Among them, facilities, leisure time, interests, habits of the rate selected in the top three, visible, inadequate facilities, primitive conditions, lack of leisure time, sports no interest, not often participate in physical activity habits, is restricting urban residents to participate in sports activities are important factors. In contrast, the activity of the necessary facilities, although also has changed, but still far can not meet the needs of people at this stage of sports activities, and thus become the main factor restricting people to participate in sports 
activities.

Table 4Influencing Factors in the survey

\begin{tabular}{|c|c|c|}
\hline & Frequency & Sequence \\
\hline Space and Facilities & 307 & 1 \\
\hline Spare Time & 251 & 2 \\
\hline Interest and Habit & 179 & 3 \\
\hline Health Situation & 154 & 4 \\
\hline Instructors & 153 & 6 \\
\hline Economic Circumstances & 87 & 5 \\
\hline Others & 32 & 7 \\
\hline
\end{tabular}

\section{Countermeasures Research}

Increase Propaganda Power. Sports is an important part of civilization, science and healthy life style, and the development of leisure sports. Department of China's traditional concepts of life and the quality of modern life and the quality of people's physical health level of improvement, the relevant government departments at all levels should increase the promotion of sports, to make full use of radio and other media are simple guide the publicity, so that the masses in the leisure time to actively participate in the sports fitness entertainment activities. In real life, the intense competition and fast-paced way of life makes people's body and mind are in a state of tension, there is an urgent need to find ways to release the pressure, through participation in recreational sports activities to release the pressure is undoubtedly the best way. And the combination of mass media and sports to make their own entertainment functions are fully excavated and further extended. Through the communication of the media, the people's potential energy will lead to the physical and mental state of leisure and entertainment, so that people's emotions can be vent, the soul is purified, the inner world is more abundant. At the same time, it can guide people to take an active part in all kinds of leisure and recreational sports, through the sports will be liberated from the pressure of people, to reduce the physical and psychological tension and depression.

Strengthen Stadium Construction. Sports venues and facilities play an important role in the development of leisure sports activities. It is the carrier of people's leisure sports activities, and is the premise and guarantee for the development of leisure sports activities. High quality sports venues and facilities can not only meet the requirements of urban residents engaged in leisure sports activities, but also to attract more and more people to participate in leisure sports activities. A large number of studies show that the main limitation of urban residents' participation in leisure sports is the lack of necessary sports facilities. To this end, in recent years, China's government has increased the intensity of sports venues and facilities, through the use of sports lottery public welfare fund in the community and park construction of sports venues and facilities and fitness equipment. Relevant government departments at all levels should increase the construction of public stadiums and gymnasiums, built some necessary fitness facilities, play to the function of the government management, use of social forces, absorb all funds, participate in sports investment and business, promote the development of related industries. Sports venues and facilities should be incorporated into the urban construction planning, and set aside the necessary land area, to ensure that the legislation to be strictly imposed on the use of physical and sports. The construction of the city should carry out leisure sports as an essential content, where the new residential district must be equipped with the appropriate sports venues. Those who have sports facilities and enterprises and institutions and schools, to give full play to the role of existing sports venues, to provide convenience for sports.

Organize Sports Activities. Sports big country to sports power forward, the development of mass sports is to lay the foundation for the development of competitive sports, only the masses of sports to carry out a good foundation to play a strong competitive sports talent selection to have enough space. Mass sports activities to close to the needs of the masses to carry out, to meet the needs of different groups of sports training needs and to carry out various kinds of sports activities, 
and more people have the opportunity to participate in sports activities, when people adapt to the sports activities to bring benefits to generate interest, will have to adhere to the possible involvement. It is hoped that the sports grassroots departments in the organization and planning to carry out the activities of the study and consider the needs of different urban residents, to carry out more suitable for different regions, different gender, different age groups. At the same time hope that such as the community games and other events to carry out a lot of competition, the city, District, street level linkage, regular games, to provide a platform for community residents to participate. We hope that sports will fully mobilize the enthusiasm of all levels of society, and actively organize sports activities for the city residents to participate in sports activities to create a good atmosphere. Hand, on the basis of macroeconomic regulation and control of the state of sports and leisure time for guarantee work, community leisure sports 1 hour; on the other hand, on the basis of the contents of the existing activities can through the mining of traditional sports project to meet the public demand.

Cultivate Professinal Talents. Sports development needs a lot of with specialized knowledge and skills of the professionals to provide support, including: sports management personnel, through the reasonable management can enhance leisure sports industry vitality, active sports consumption market; sports scientific research personnel, through a variety of research results, scientific guidance of leisure sports development; social sports instructors, effective organization to guide people to leisure and fitness activities. Strengthening the cultivation of professional talents, in the same time, we should play the role of the main channel of physical education colleges and universities. Some colleges and universities set up sports major, utilize the advantage of its educational resources, train all kinds of high quality professional talents. Strengthen the construction of social sports instructor team, no professional guidance is a lot of urban residents to participate in sports activities. At present, the number of social sports instructors is less, some people do not have the actual guidance qualifications and experience, just to meet the requirements of the training of higher level units. At present, the training form and content of the social sports instructors are very different from the practical guidance. We should change the current situation of social sports instructors and improve the form and content of training as soon as possible.

\section{Acknowledgements}

This research is financially supportedby 2016 Fundamental Research Funds for Heilongjiang Provincial Education Department(Grant No.2016-KYYWF-0898.). The name of the project is "The Research on Participation Analysis and Service Supply of Sports of Heilongjiang Citizens”.

\section{References}

[1] Li Bin, Tang Xun, Huang Pinpin, Journal of Southwest China Normal University (Natural Science Edition), Vol. 38(2013) No 2, p.127-132

[2] Zhao Junhong, Fang Min, Journal of Sports and Science, Vol. 34(2013) No 4, p.112-115

[3] Zhong Hua, Zhang Guoqing, Reformation \& Strategy, Vol. 26(2010) No 7, p.125-127

[4] Li Ye, BianZhenwu, Modern Business Trade Industry, Vol. 29(2016) No 17, p.25-26 